\title{
Diversity of Invertebrate Discards in Small and Medium Scale Aegean Sea Fisheries
}

\author{
Eleni Voultsiadou*, Constantinos Fryganiotis, Maria Porra, Panagiotis Damianidis and \\ Chariton-Charles Chintiroglou*
}

Department of Zoology, School of Biology, Aristotle University of Thessaloniki, 54124 Thessaloniki, Greece

\begin{abstract}
In the present work, an attempt is made to evaluate and compare the diversity of invertebrate discards from small (gill/trammel nets) and medium (otter trawling) scale fisheries in Thermaikos Gulf (North Aegean Sea), which is a major fishing area of the eastern Mediterranean but still unexplored for its benthic community structure. Sampling was performed under normal fishing activities in 21 otter trawl hauls and 62 set nets operations. One hundred thirty-two invertebrate species were altogether discarded in medium and small scale fishery over the study area, classified in 16 classes and 7 phyla. Small scale fisheries yielded almost $70 \%$ of the total invertebrate diversity, while otter trawling only $50 \%$. Molluscs were the most diverse group in both fisheries followed by crustaceans and echinoderms. Multivariate analysis revealed a clear differentiation in discard species composition between the two fisheries reflecting different benthic habitats. According to the species composition of the discards certain benthic biocoenoses were identified. The Coastal terrigenous muds biocoenosis prevailed in medium scale fishing areas, accompanied by the Coastal detritic, Muddy detritic, and Bathyal mud biocoenoses. Several plant-dominated communities (mostly those of Infralittoral photophilus algae, Circalittoral sciaphilus algae, and Posidonia meadows), were recognized through small scale fishery discards. The higher diversity of the latter was attributed to the great variety of habitats and established communities in the relevant fishing grounds. These results show that invertebrate discards could contribute background data for monitoring the complex benthic system.
\end{abstract}

Keywords: Fisheries, Discards, Benthic communities, Greece, Eastern Mediterranean, Thermaikos Gulf.

\section{INTRODUCTION}

The current estimate of global marine fisheries by-catch approximates $40 \%$ of the total catch, not taking into account the invertebrates, due to lack of sufficient data [1]. Invertebrate by-catch usually includes benthic representatives of animal taxa such as molluscs, crustaceans, echinoderms and other taxa. Some of them, despite their commercial value, are occasionally discarded for various reasons [2], while others have no economic value at all. In any case, the removal of benthic community components from the sea bottom and their transportation to different areas, depths, or habitats might be of particular importance for the benthic ecosystem [3]. Moreover, epifaunal macro-zoobenthic communities, which are severely disturbed by certain fishing activities, seem to play a key role in structuring demersal fish assemblages [4]. Therefore, the relationships between benthic invertebrate and fish communities have been set as priority issues for the development of spatial management units, if a holistic ecosystem management approach is to be promoted [5].

Despite the importance of the discarded invertebrates for the benthic ecosystem and the fact that they may account for more than $60 \%$ of the total discards [3] few studies have

*Address correspondence to these authors at the Department of Zoology, School of Biology, Aristotle University of Thessaloniki, 54124 Thessaloniki, Greece; Tel: +302310998321; Fax: +302310998269; E-mail: elvoults@bio.auth.gr; Tel: +302310998405; E-mail: chintigl@bio.auth.gr addressed the problem of their diversity at the species level. From this point of view, and considering their impact on the sea bottom communities, medium scale fisheries, i.e. trawling practices, have been studied in Southern Portugal [6] and western Mediterranean [7]. On the contrary, small scale or artisanal fisheries, although accounting for about 50 $\%$ of the fish consumed by humans [8], have only recently received attention regarding their invertebrate discard diversity, in the Portuguese coasts [3,9].

The Hellenic fisheries is of multi-gear and multi-species nature, the small scale fishery representing $45 \%$ and the trawl fishery $27 \%$ of the total annual catch [10]. Various publications have addressed the discard issue from different aspects focusing on the discarded fish species (e.g. 11, 12, 13]. Benthic invertebrate discards have not been paid special attention and there is practically only one publication [14] listing crustacean and cephalopod discards from some Hellenic marine areas.

The fishing area of Thermaikos Gulf is among the most productive in the Eastern Mediterranean, the annual fishing catch with both medium and small scale fisheries reaching $24 \%$ of Greek fisheries landings [10]. It has been considered as a distinct fishing sub-area of the Aegean Sea according to the composition of fish assemblages [15]. However, information on the structure of sublittoral benthic communities in Thermaikos Gulf is still scarce $[16,17]$.

Taking into account i) the significance of Thermaikos marine area for the Hellenic fisheries and its prolonged exploitation, ii) the absence of data on the invertebrate discards 
and the limited information on the composition of benthic invertebrate communities in this area, and iii) the worldwide scarce data on the discards of small scale fisheries, a first attempt is made herein to record and compare the invertebrate diversity discarded in medium and small scale fisheries in this major fishing area of the Aegean Sea. This study further aims at examining the possible utility of discard diversity as background information for evaluating community structure and distribution in the complex benthic environment.

\section{MATERIALS AND METHODOLOGY}

Sampling of discards in small and medium scale fisheries was carried out in different areas of Thermaikos Gulf (Fig. 1). Sampling sites are illustrated in the map of Fig. (1), on which sediment characteristics were depicted according to Karageorgis \& Anagnostou [18].
Small scale fisheries (Ssf) sampling was carried out in 26 fishing vessels at 7 seven fishing ports (stations: 22 Platamonas at 7-41 m, 23 Katerini at 20-46 m, 24 Litochoro at 6-40 m, 25 Kalamaria at 9-30 m, 26 Michaniona at 3-34 $\mathrm{m}, 27$ Kallikratia at $37-63 \mathrm{~m}$ and 28 Potidea at 4-45 m) in Thermaikos Gulf (Fig. 1), between September 2007 and May 2008. The sampling vessels fished with trammel nets, gill nets, or both types, usually unselectively, seasonally targeting mainly the common sole, Solea solea, and the stripped red mullet, Mullus surmuletus. Nets were 1000$7000 \mathrm{~m}$ in length, $0.7-2.0 \mathrm{~m}$ in height, had a mesh size of 17$50 \mathrm{~mm}$, and were anchored at depths of 3-63 m for 1 to 24 hours. In total 62 nets thrown in 7 areas in the vicinity of the above mentioned fishing ports were examined. After the selection of the commercial catch, the discards were sent to the laboratory preserved in $10 \%$ formalin.

In order to assess how well the samples taken represented the invertebrate diversity in the discards, the cumulative

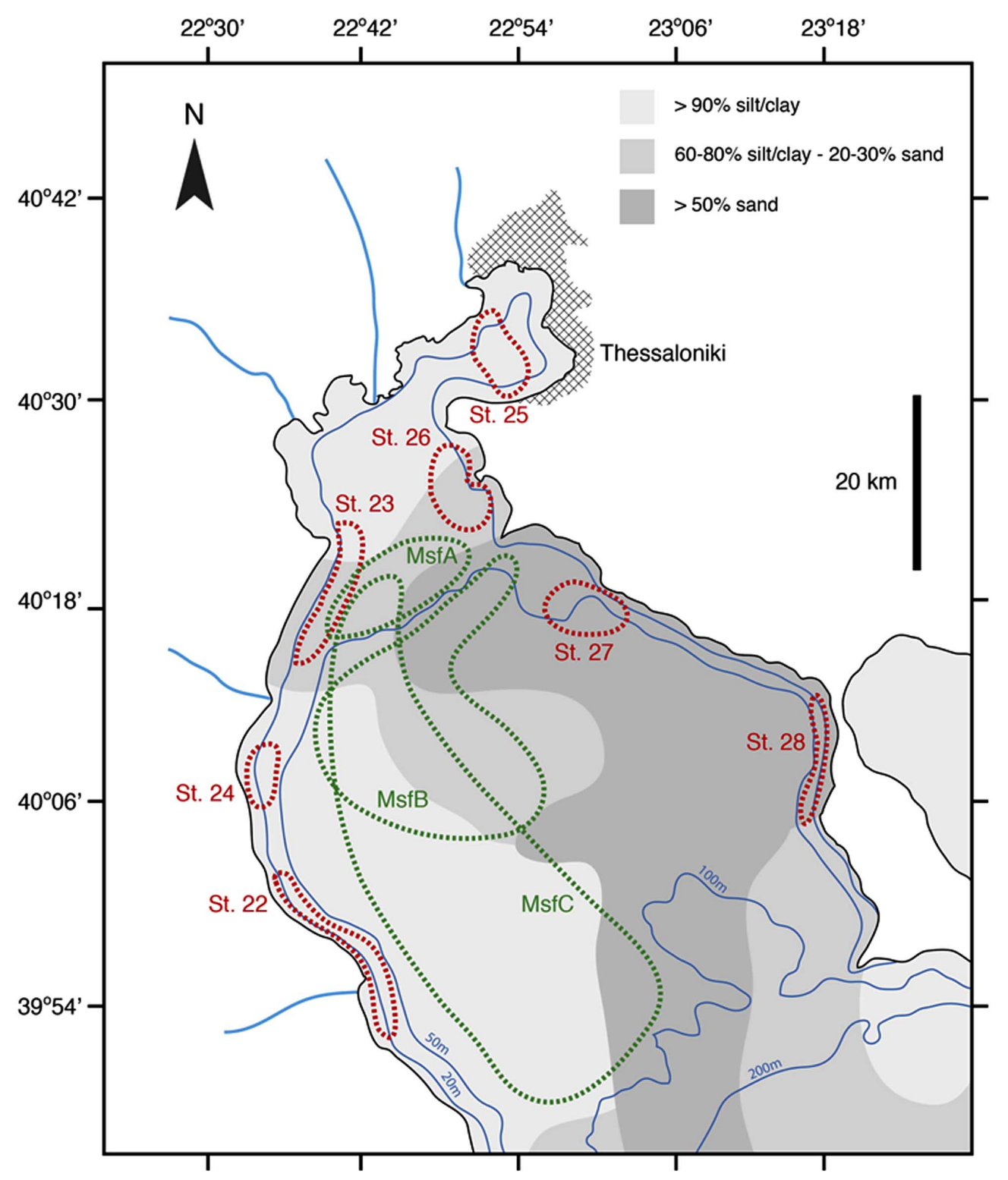

Fig. (1). Map of Thermaikos Gulf showing sampled areas. Green dotted lines indicate Medium scale fishery (Msf) stations (trawled bottoms); red dotted lines indicate Small scale fishery stations (gill/trammel nets fishing grounds). 
number of species collected was plotted against the number of nets examined $\left(y=27.95 \operatorname{Ln}[x]-28.68, \mathrm{R}^{2}=0.839\right)$. Then, the nets thrown in the same fishing area were merged into a single set (totally 7 sets).

Medium scale fisheries (Msf) sampling took place onboard commercial otter trawlers between October 2005 and April 2006 (the otter trawling is permitted from October to May all over the Greek territory) only in the outer part of Thermaikos Gulf, since otter trawling is prohibited in Thessaloniki Bay throughout the year. Among the main target species were the shrimps Melicertus kerathurus and Parapenaeus longirostris. Three commercial otter trawlers were used all stationed at the fishing port of Michaniona. Totally 21 hauls (stations) were surveyed (Fig. 1): Stations 1-8 in the northern part of the Gulf, at 33-48 m (Autumn 2005) collectively labelled as MsfA; stations 9-14 in the northwest part, at 46-88 m (Winter 2006), labelled MsfB; and stations 15-21 in the western part, at 42-95 $\mathrm{m}$ (Spring 2006), labelled as MsfC. Sampling was performed under normal fishing activities and included the following steps: i) at first large-sized $\left(>10 \mathrm{~cm}^{3}\right)$ specimens, e.g. of bivalves, sponges and gastropods, were collected; ii) the total catch volume was roughly estimated by computing the trawl cone volume iii) catches were sorted for commercial species and the non-commercial by-catch was kept apart, but not thrown away; iv) since the catches were typically large, a subsample was randomly taken, using a large shovel until two plastic basins, 401 each, were filled. In this way, about 1/10 of the total discards was kept in $10 \%$ formalin for sorting and identification. Totally, more than 8001 of discards were examined. Plotted against the number of hauls, the cumulative number of discarded invertebrate species showed that more than $80 \%$ of the discarded abundance was obtained $\left(y=10.99 \operatorname{Ln}[x]+27.73, \mathrm{R}^{2}=0.985\right)$.

Multivariate analyses i.e. cluster and multidimensional scaling (MDS) were performed on presence/absence data based on the Bray-Curtis similarity, in order to analyse the similarity among the sampling stations. SIMPER analysis indicated the percentage contribution of each species to the overall similarity within stations. For the above analyses the Primer package [19] was used.

The assignment of discarded species to benthic biocoenoses was made mainly according to Peres and Picard [20], Augier [21], and Aguilar et al., [22].

\section{RESULTS}

One hundred thirty-two invertebrate species (Table 1) were altogether discarded in Msf and Ssf over the study area, classified in 16 classes and 7 phyla (Fig. 2). Molluscs were the most diverse group (39\% of the total number of species) followed by crustaceans (23\%) and echinoderms (17\%). Other taxa provided lower contributions in terms of species numbers: cnidarians $8 \%$, sponges $6 \%$, ascidians $5 \%$ and annelids $2 \%$. When each fishery type was examined separately, a similar ranking was observed. Among molluscs, gastropods and bivalves dominated with benthic representatives, while cephalopods included benthic and 3 pelagic species. Among echinoderms, holothuroids and asteroids were the most diverse.
Table 1. Invertebrate Species Caught by Medium (Msf) and Small Scale (Ssf) Fisheries in Thermaikos Gulf and their Frequency of Appearance (F) in Sampling Stations (Frequencies $>\mathbf{8 0} \%$ in Bold)

\begin{tabular}{|c|c|c|c|}
\hline \multirow{2}{*}{ Species } & \multicolumn{3}{|c|}{ F (\%) } \\
\hline & Msf & Ssf & Total \\
\hline \multicolumn{4}{|l|}{ PORIFERA } \\
\hline \multicolumn{4}{|l|}{ Demospongiae } \\
\hline Aplysina aerophoba (Nardo, 1843) & 19.5 & 42.86 & 25.00 \\
\hline Axinella cannabina (Esper, 1794) & - & 14.28 & 3.57 \\
\hline Chondrosia reniformis (Nardo, 1847) & - & 28.57 & 7.14 \\
\hline Cliona viridis (Schmidt, 1862) & - & 14.28 & 3.57 \\
\hline Petrosia (Petrosia) ficiformis (Poiret, 1789) & 38.10 & - & 28.57 \\
\hline Scalarispongia scalaris (Schmidt, 1862) & 9.52 & - & 7.14 \\
\hline Tethya aurantium (Pallas, 1766) & 4.76 & - & 3.57 \\
\hline Ulosa stuposa (Esper, 1794) & 9.52 & - & 7.14 \\
\hline \multicolumn{4}{|l|}{ CNIDARIA } \\
\hline \multicolumn{4}{|l|}{ Anthozoa } \\
\hline Alcyonium palmatum (Pallas, 1766) & 100.00 & 28.57 & 82.14 \\
\hline Calliactis parasitica (Couch, 1838) & 52.38 & 100.00 & 64.28 \\
\hline Cerianthus membranaceus (Spallanzani, 1784) & 4.76 & - & 3.57 \\
\hline Cladocora caespitosa (Linnaeus, 1758) & - & 28.57 & 7.14 \\
\hline $\begin{array}{l}\text { Crassophyllum thessalonicae } \\
\text { (Vafidis\&Koukouras,1991) }\end{array}$ & 19.05 & - & 14.28 \\
\hline Funiculina quadrangularis (Pallas, 1766) & 4.76 & - & 3.57 \\
\hline Pennatula rubra (Ellis, 1761) & 95.24 & - & 71.43 \\
\hline Pteroides griseum (Bohadsch 1761) & 28.57 & - & 21.43 \\
\hline Sagartiogeton undatus (Müller, 1778) & 38.10 & 14.28 & 32.14 \\
\hline Veretillum cynomorium (Pallas, 1766) & 28.57 & 14.28 & 25.00 \\
\hline \multicolumn{4}{|l|}{ ANNELIDA } \\
\hline \multicolumn{4}{|l|}{ Clitellata } \\
\hline Pontobdella muricata (Linnaeus, 1758) & 19.05 & 14.28 & 17.86 \\
\hline \multicolumn{4}{|l|}{ Polychaeta } \\
\hline Aphrodita aculeata (Linnaeus, 1758) & 47.62 & - & 35.71 \\
\hline \multicolumn{4}{|l|}{ MOLLUSCA } \\
\hline \multicolumn{4}{|l|}{ Polyplacophora } \\
\hline Chiton (Rhyssoplax) olivaceus (Spengler, 1797) & - & 14.28 & 3.57 \\
\hline \multicolumn{4}{|l|}{ Gastropoda } \\
\hline Aplysia sp. & - & 14.28 & 3.57 \\
\hline Aporrhais pespelecani (Linnaeus, 1758) & 61.90 & 100.00 & 71.43 \\
\hline Aporrhais serresianus (Michaud, 1828) & - & 28.57 & 7.14 \\
\hline Euspira guillemini (Payraudeau, 1826) & - & 14.28 & 3.57 \\
\hline Fasciolaria lignaria (Linnaeus, 1758) & - & 14.28 & 3.57 \\
\hline Buccinulum corneum (Linnaeus, 1758) & - & 14.28 & 3.57 \\
\hline Cerithium vulgatum (Bruguière, 1792) & - & 71.43 & 17.86 \\
\hline Diodora gibberula (Lamarck, 1822) & - & 14.28 & 3.57 \\
\hline Bolinus brandaris (Linnaeus, 1758) & 76.19 & 100.00 & 82.14 \\
\hline Bolma rugosa (Linnaeus, 1767) & - & 14.28 & 3.57 \\
\hline $\begin{array}{l}\text { Hexaplex (Trunculariopsis) trunculus } \\
\text { (Linnaeus, 1758) }\end{array}$ & 14.29 & 100.00 & 35.71 \\
\hline Galeodea echinophora (Linnaeus, 1758) & 95.24 & 85.71 & 92.86 \\
\hline
\end{tabular}




\begin{tabular}{|c|c|c|c|}
\hline \multirow{2}{*}{ Species } & \multicolumn{3}{|c|}{ F (\%) } \\
\hline & Msf & Ssf & Total \\
\hline Fusinus rostratus (Olivi, 1792) & - & 14.28 & 3.57 \\
\hline Fusinus syracusanus (Linnaeus, 1758) & - & 28.57 & 7.14 \\
\hline Nassarius incrassatus (Ström, 1768) & - & 28.57 & 7.14 \\
\hline Nassarius reticulatus (Linnaeus, 1758) & - & 14.28 & 3.57 \\
\hline Ocenebra erinaceus (Linnaeus, 1758) & 4.76 & 14.28 & 7.14 \\
\hline Pleurobranchaea meckelii (Leue, 1813) & - & 14.28 & 3.57 \\
\hline Spondylus gaederopus (Linnaeus, 1758) & - & 14.28 & 3.57 \\
\hline Tonna galea (Linnaeus, 1758) & 33.33 & 71.43 & 42.86 \\
\hline Turritella communis (Risso, 1826) & 71.43 & 71.43 & 71.43 \\
\hline \multicolumn{4}{|l|}{ Bivalvia } \\
\hline Acanthocardia echinata (Linnaeus, 1758) & 100.00 & - & 75.00 \\
\hline Acanthocardia spinosa (Lightfoot, 1786) & - & 14.28 & 3.57 \\
\hline Acanthocardia tuberculata (Linnaeus, 1758) & - & 14.28 & 3.57 \\
\hline Aequipecten opercularis (Linnaeus, 1758) & 14.29 & - & 10.71 \\
\hline Anadara diluvii (Lamarck, 1805) & 9.52 & - & 7.14 \\
\hline Anadara corbuloides (Monterosato, 1878) & - & 14.28 & 3.57 \\
\hline Arca noae (Linnaeus, 1758) & - & 14.28 & 3.57 \\
\hline Atrina pectinata (Linnaeus, 1767) & 80.95 & - & 60.71 \\
\hline Callista chione (Linnaeus, 1758) & - & 14.28 & 3.57 \\
\hline Flexopecten flexuosus (Poli, 1795) & - & 14.28 & 3.57 \\
\hline Glossus humanus (Linnaeus, 1758) & 33.33 & - & 25.00 \\
\hline Mimachlamys varia (Linnaeus, 1758) & 28.57 & 28.57 & 28.57 \\
\hline Modiolus barbatus (Linnaeus, 1758) & - & 14.28 & 3.57 \\
\hline Modiolus sp. & - & 14.28 & 3.57 \\
\hline Ostrea edulis (Linnaeus, 1758) & - & 28.57 & 7.14 \\
\hline Pecten jacobaeus (Linnaeus, 1758) & 4.76 & 14.28 & 7.14 \\
\hline Pseudamussium clavatum (Poli, 1795) & - & 14.28 & 3.57 \\
\hline Scrobicularia plana (da Costa, 1778) & & 14.28 & 3.57 \\
\hline Thracia pubescens (Pulteney, 1799) & 4.76 & - & 3.57 \\
\hline Venus verrucosa (Linnaeus, 1758) & - & 14.28 & 3.57 \\
\hline \multicolumn{4}{|l|}{ Cephalopoda } \\
\hline Alloteuthis media (Linnaeus, 1758) & 38.10 & - & 28.57 \\
\hline Alloteuthis subulata (Lamarck, 1798) & 28.57 & - & 21.43 \\
\hline Eledone cirrhosa (Lamarck, 1798) & 14.29 & - & 10.71 \\
\hline Eledone moschata (Lamarck, 1798) & - & 28.57 & 7.14 \\
\hline Illex coindetii (Vérany, 1839) & 47.62 & - & 35.71 \\
\hline Rondeletiola minor (Naef, 1912) & 33.33 & - & 25.00 \\
\hline Sepia elegans (Blainville, 1827) & 85.71 & - & 64.28 \\
\hline Sepia officinalis (Linnaeus, 1758) & 23.81 & 28.57 & 25.00 \\
\hline Sepiola intermedia (Naef, 1912) & 9.52 & - & 7.14 \\
\hline Sepiola robusta (Naef, 1912) & 19.05 & - & 14.28 \\
\hline Todarodes sagittatus (Lamarck, 1798) & - & 14.28 & 3.57 \\
\hline \multicolumn{4}{|l|}{ CRUSTACEA } \\
\hline \multicolumn{4}{|l|}{ Maxillopoda } \\
\hline Scalpellum scalpellum (Linnaeus, 1767) & 14.29 & - & 10.71 \\
\hline \multicolumn{4}{|l|}{ Malacostraca } \\
\hline Anilocra physodes (Linnaeus, 1758) & - & 14.28 & 3.57 \\
\hline Dardanus arrosor (Herbst, 1796) & - & 14.28 & 3.57 \\
\hline Dardanus calidus (Risso, 1827) & - & 42.86 & 10.71 \\
\hline
\end{tabular}

\begin{tabular}{|c|c|c|c|}
\hline \multirow[t]{2}{*}{ Species } & \multicolumn{3}{|c|}{ F (\%) } \\
\hline & Msf & Ssf & Total \\
\hline Dromia personata (Linnaeus, 1758) & - & 14.28 & 3.57 \\
\hline Ethusa mascarone (Herbst, 1785) & - & 42.86 & 10.71 \\
\hline Galathea strigosa (Linnaeus, 1761) & - & 14.28 & 3.57 \\
\hline Goneplax rhomboides (Linnaeus, 1758) & 61.90 & 85.71 & 67.86 \\
\hline Distolambrus maltzami (Miers, 1881) & - & 28.57 & 7.14 \\
\hline Calappa granulata (Linnaeus, 1758) & - & 14.28 & 3.57 \\
\hline Carcinus aestuarii (Nardo, 1847) & - & 14.28 & 3.57 \\
\hline Ilia nucleus (Linnaeus, 1758) & - & 28.57 & 7.14 \\
\hline Liocarcinus corrugatus (Pennant, 1777) & - & 28.57 & 7.14 \\
\hline Liocarcinus depurator (Linnaeus, 1758) & 100.00 & 71.43 & 92.86 \\
\hline Macropodia longirostris (Fabricius, 1775) & - & 71.43 & 17.86 \\
\hline Maja crispata (Risso, 1827$)$ & - & 42.86 & 10.71 \\
\hline Maja goltziana (d'Oliviera, 1888) & - & 14.28 & 3.57 \\
\hline Maja squinado (Herbst, 1788) & - & 42.86 & 10.71 \\
\hline Medorippe lanata (Linnaeus, 1767) & 95.24 & 71.43 & 89.28 \\
\hline Melicertus kerathurus (Forskål, 1775) & 66.67 & - & 50.00 \\
\hline Munida sarsi (Huus, 1935) & 33.33 & - & 25.00 \\
\hline Nephrops norvegicus (Linnaeus, 1758) & 28.57 & - & 21.43 \\
\hline Paguristes eremita (Linnaeus, 1767) & - & 100.00 & 25.00 \\
\hline Pagurus cuanensis (Bell, 1845) & - & 57.14 & 14.28 \\
\hline Pagurus excavatus (Herbst, 1791) & 47.62 & 85.71 & 57.14 \\
\hline Parapenaeus longirostris (Lucas, 1846) & 100.00 & 14.28 & 78.57 \\
\hline Pilumnus hirtellus (Linnaeus, 1761) & - & 57.14 & 14.28 \\
\hline Pisidia longimana (Risso, 1816) & - & 28.57 & 7.14 \\
\hline Sicyonia carinata (Brünnich, 1768) & 28.57 & - & 21.43 \\
\hline Squilla mantis (Linnaeus, 1758) & 100.00 & 100.00 & 100.00 \\
\hline \multicolumn{4}{|l|}{ ECHINODERMATA } \\
\hline \multicolumn{4}{|l|}{ Crinoidea } \\
\hline Antedon mediterranea (Lamarck, 1816) & - & 14.28 & 3.57 \\
\hline \multicolumn{4}{|l|}{ Echinoidea } \\
\hline Arbacia lixula (Linnaeus, 1758) & - & 14.28 & 3.57 \\
\hline $\begin{array}{l}\text { Brissopsis atlantica var. mediterranea } \\
\text { (Mortensen, 1907) }\end{array}$ & 4.76 & - & 3.57 \\
\hline Sphaerechinus granularis (de Lamarck, 1816) & - & 71.43 & 17.86 \\
\hline Psammechinus microtuberculatus (Heller, 1868) & - & 14.28 & 3.57 \\
\hline Paracentrotus lividus (Lamarck, 1816) & - & 71.43 & 17.86 \\
\hline \multicolumn{4}{|l|}{ Holothurioidea } \\
\hline Holothuria (Holothuria) tubulosa (Gmelin, 1790) & 23.81 & 42.86 & 28.57 \\
\hline Holothuria (Roweothuria) poli (Delle Chiaje, 1823) & 9.52 & - & 7.14 \\
\hline Leptopentacta elongata (Düben \& Koren, 1846) & 38.10 & - & 28.57 \\
\hline Leptopentacta tergestina (M. Sars, 1857) & 9.52 & - & 7.14 \\
\hline Ocnus planci (Brandt, 1835) & 42.86 & 14.28 & 35.71 \\
\hline Ocnus sp. & - & 14.28 & 3.57 \\
\hline Parastichopus regalis (Cuvier, 1817) & 66.67 & 14.28 & 53.57 \\
\hline \multicolumn{4}{|l|}{ Asteroidea } \\
\hline Astropecten aranciacus (Linnaeus, 1758) & 14.29 & 71.43 & 28.57 \\
\hline $\begin{array}{l}\text { Astropecten irregularis pentacanthus } \\
\text { (Delle Chiaje, 1827) }\end{array}$ & 52.38 & 71.43 & 57.14 \\
\hline Astropecten jonstoni (Delle Chiaje, 1827) & - & 28.57 & 7.14 \\
\hline
\end{tabular}




\begin{tabular}{|l|c|c|c|}
\hline \multirow{2}{*}{ Species } & \multicolumn{3}{|c|}{ F (\%) } \\
\cline { 2 - 4 } & Msf & Ssf & Total \\
\hline \hline Astropecten spinulosus (Philippi, 1837) & - & $\mathbf{8 5 . 7 1}$ & 21.43 \\
\hline Chaetaster longipes(Retzius, 1805) & - & 14.28 & 3.57 \\
\hline Echinaster (Echinaster) sepositus (Retzius, 1783) & - & 14.28 & 3.57 \\
\hline Marthasterias glacialis (Linnaeus, 1758) & 42.86 & 14.28 & 35.71 \\
\hline \multicolumn{1}{|c|}{ Ophiuroidea } & & & \\
\hline Ophioderma longicauda (Bruzelius, 1805) & - & 14.28 & 3.57 \\
\hline Ophiothrix fragilis (Abildgaard, 1789) & - & 28.57 & 7.14 \\
\hline & & & \\
\hline \multicolumn{1}{|c|}{ AsDATA } & & & \\
\hline Ascidia mentula (Müller, 1776) & - & 14.28 & 3.57 \\
\hline Ascidia sp. & 52.38 & - & 39.28 \\
\hline Microcosmus sabatieri Roule, 1885 & 42.86 & - & 32.14 \\
\hline Microcosmus sp. & - & 71.43 & 17.86 \\
\hline Molgula sp. & - & 14.28 & 3.57 \\
\hline Phallusia mamillata (Cuvier, 1815) & 66.67 & 71.43 & 67.86 \\
\hline Styela sp. & 66.67 & 57.14 & 64.28 \\
\hline
\end{tabular}

Msf and Ssf accounted for 65 and 97 discarded species respectively. Differences in the qualitative composition of the discarded invertebrates between the two fishery types are obvious (Table 1, Fig. 2), since only 29 species were common to both. Certain groups, such as anthozoans, holothuroids, demosponges and ascidians were more diverse in
Msf discards. On the other hand, crustaceans, gastropods and asteroids were more diverse in the Ssf.

Fishery type was the main factor determining ordination of sampling stations in both hierarchical clustering and MDS analyses (Fig. 3a, b). Two different groups were identified at a similarity level of $31 \%$, one of which included the small scale fishery stations ( $\mathrm{Ssf}$ ) and another comprising the medium scale fishery stations (Msf). In the latter, three groups were recognized (58.6\% similarity). Each of these groups included the hauls sampled in the three different areas-depths of the outer Thermaikos Gulf and in different seasons.

As shown by SIMPER analysis, the average similarity among the stations of the three groups of Msf ranged from 69 to $76 \%$, while small differences in the numbers of species among the three groups of Msf stations were observed: 54 species in MsfA, 46 species in MsfB and 49 species in MsfC. Much lower average similarity (50\%) was observed among the Ssf stations; here, species richness varied among the sampling stations, from 20 species $(21 \%$ of the total Ssf species richness) in station 25 (Kalamaria) to 44 species $(48 \%)$ in station 27 (Kallikrateia), with all other stations having intermediate values of $30-40 \%$. According to their invertebrate species composition, stations 25 and 28 were separated from the remaining Ssf stations (Fig. 3b).

Considering both fishery types, 15 species were present in more than $50 \%$ of the stations (Table 1), thus characterized as common species in Thermaikos Gulf, while one, Squilla mantis, was omnipresent. The Msf and Ssf station

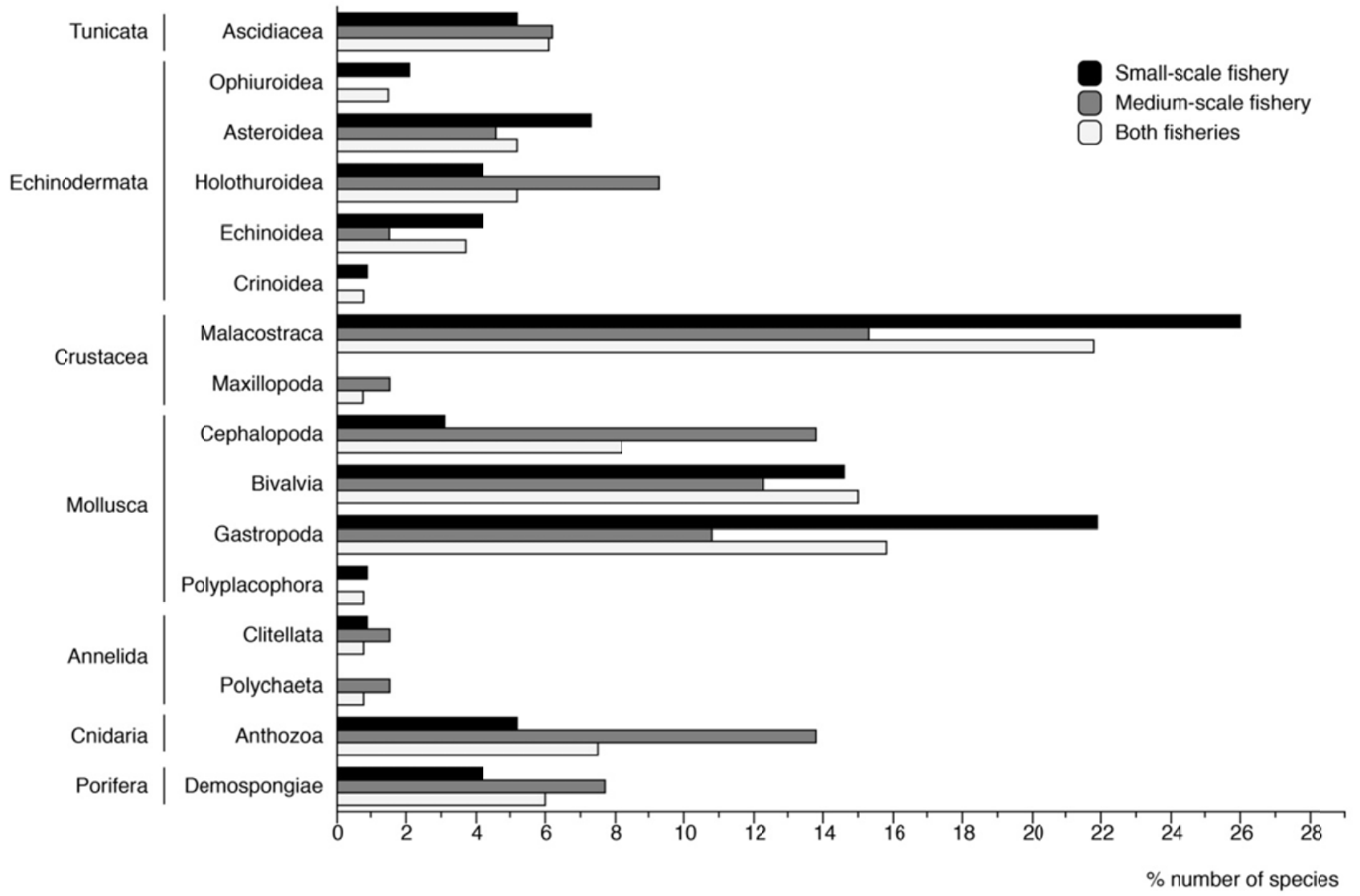

Fig. (2). Number of species of invertebrate groups discarded in Thermaikos Gulf. 
(a)

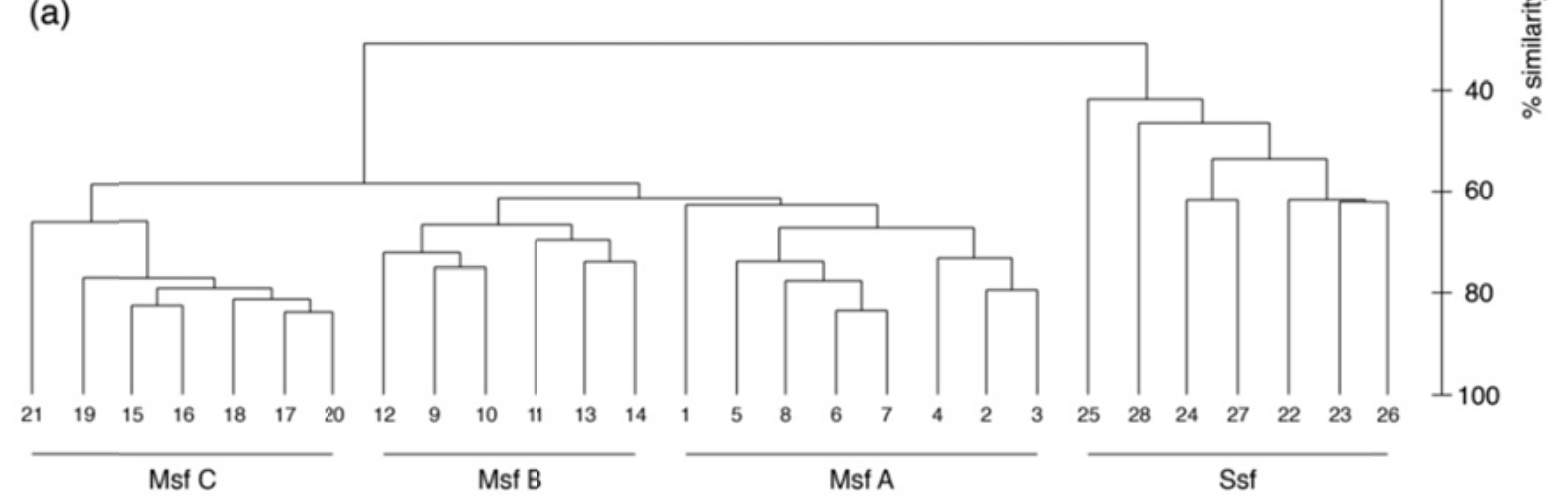

(b)

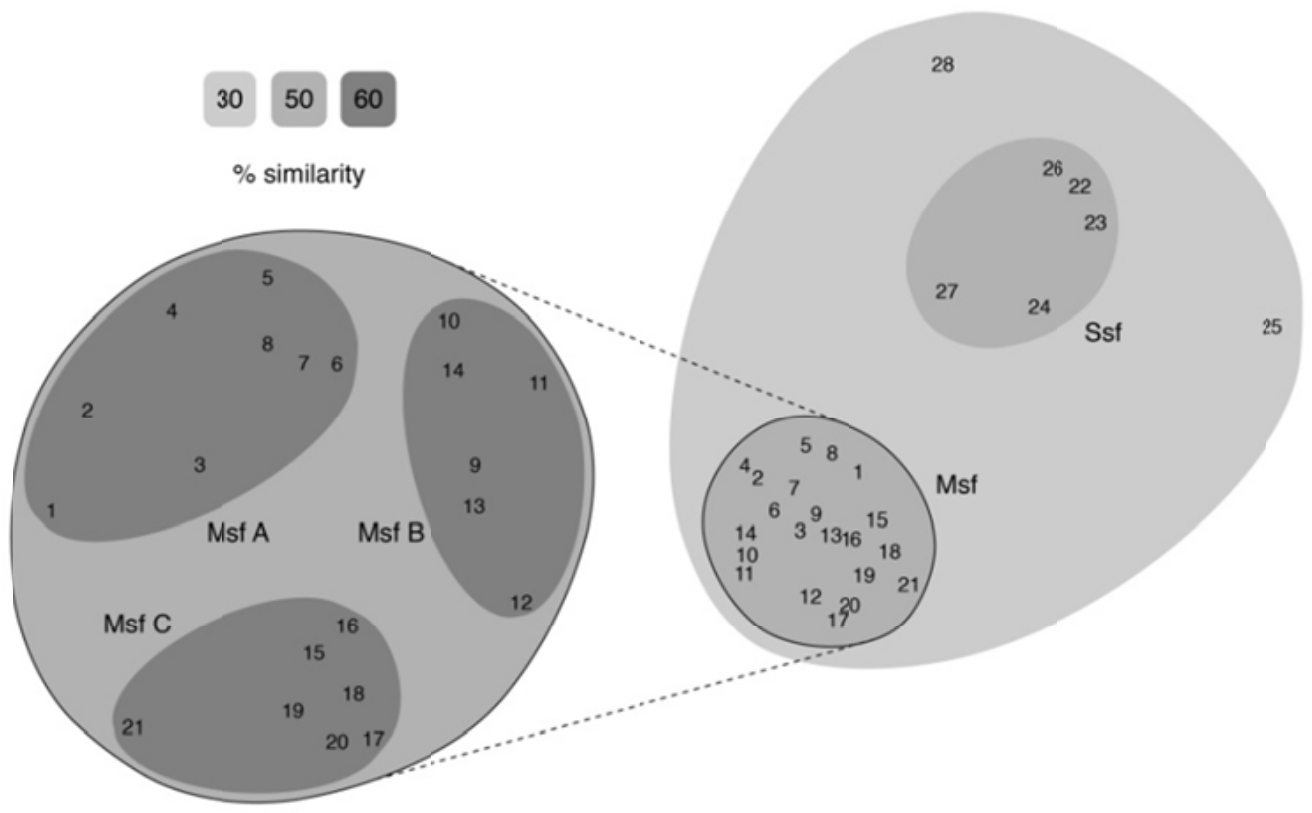

Fig. (3). Similarity among fishing areas in Thermaikos Gulf based on diversity of invertebrate discards in Medium (Msf) and Small scale (Ssf) fisheries. The increasingly dark shades join areas of increasing \% similarity. For labelling see Methodology.

groups were clearly discriminated on the basis of the species responsible for in-group similarity. In Ssf stations the species responsible for $50 \%$ in-group similarity were Aporrhais pespelecani, Bolinus brandaris, Calliactis parasitica, Hexaplex trunculus, Paguristes eremita, Squilla mantis, Goneplax rhomboides, Pagurus excavatus, Galeodea echinophora, Astropecten spinulosus; the first six of them were present in all Ssf stations. On the other hand, the three Msf groups demonstrated identical species composition at a cumulative contribution level of $31-43 \%$; the species responsible for $50 \%$ in-group similarity in the three subgroups were Acanthocardia echinata, Alcyonium palmatum, Parapenaeus longirostris, Squilla mantis, Goneplax rhomboides, Liocarcinus depurator, Medorippe lanata, and Melicertus kerathurus; the first four of them were present in all Msf stations.

The great bulk of the species discarded in the Msf are among those structuring benthic biocoenoses (Table 2) typical at the soft bottoms of the lower sublittoral and bathyal zones: the Coastal detritic biocoenosis (CD), Coastal terrigenous muds biocoenosis (CTM), Muddy detritic biocoenosis (MD), and Bathyal-muds biocoenosis (BM). The invertebrate discards in the Ssf are of a more variable composition including species of both lower and upper sublittoral biocoenoses, mostly with well-developed vegetation (IPA, CCSA, HP). Finally, several of the discarded species occurring in the Msf or Ssf discards or both were eurybathic ubiquitous species (EUBUS).

\section{DISCUSSION}

A great variety of invertebrates (132 species, of which only 24 commercial) were discarded in medium and small scale fisheries in Thermaikos Gulf, one of the most intensively fished coastal areas in the Eastern Mediterranean. Molluscs, followed by crustaceans and echinoderms, dominated in both fisheries, as recently shown for trawl bycatch composition in southern Portugal [23]. Detailed comparisons with the invertebrate discard composition of other Mediterranean areas are not easy since, despite the 
Table 2. Assignment of Discarded Species to the Benthic Biocoenoses. $\mathrm{CD}=$ Coastal detritic; $\mathrm{CTM}=\mathrm{Coastal}$ terrigenous muds; MD = Muddy detritic; BM = Bathyal muds; IPA = Calm-water infralitoral photophilous algae; CCSA = Circumlittoral calm-water sciaphilous algae (in the broad sense presented by Augier, 1982, i.e. including species needing reduced light regardless of depth); EEL = Euryhaline, eurythermal lagoon communities; HP = Posidonia meadows; WGFS =Wellgraded fine sands; BGIS = Badly-graded infralittoral sands; EUBUS = Eurybathic ubiquitous species; A, B, C = stations of medium scale fishery; $22-28$ = stations of small scale fishery

\begin{tabular}{|c|c|c|c|c|c|c|}
\hline \multicolumn{7}{|c|}{ LOWER SUBLITTORAL AND BATHYAL ZONE } \\
\hline Acanthocardia echinata & $\mathrm{A}, \mathrm{B}, \mathrm{C}$ & Alcyonium palmatum & $\mathrm{A}, \mathrm{B}, \mathrm{C}, 24,27$ & \multicolumn{2}{|c|}{ Brissopsis atlantica } & $\mathrm{B}$ \\
\hline Aporrhais pespelecani & $\mathrm{A}, \mathrm{C}, 22-28$ & Ascidia mentula & 23 & \multicolumn{2}{|c|}{ Mimachlamys varia } & A,B, 25,26 \\
\hline Arca noae & 25 & Bolinus brandaris & $\mathrm{A}, \mathrm{B}, \mathrm{C}, 22-28$ & \multicolumn{2}{|c|}{ Nephrops norvegicus } & $\mathrm{C}$ \\
\hline Dardanus arrosor & 27 & Galeodea echinophora & $\mathrm{A}, \mathrm{B}, \mathrm{C}, 23-28$ & & & \\
\hline Dardanus calidus & $22,24,27$ & Goneplax rhomboides & $\mathrm{A}, \mathrm{B}, \mathrm{C}, 22,23,25-28$ & & $\mathrm{BM}$ & \\
\hline Flexopecten flexuosus & 25 & Leptopentacta elongata & $\mathrm{A}, \mathrm{B}, \mathrm{C}$ & \multicolumn{2}{|c|}{ Aporrhais serresianus } & 23,26 \\
\hline Fusinus rostratus & 25 & Leptopentacta tergestina & $\mathrm{C}$ & \multicolumn{2}{|c|}{ Funiculina quadrangularis } & $\mathrm{C}$ \\
\hline Pagurus cuanensis & $23-25,27$ & Tonna galea & $\mathrm{A}, \mathrm{C}, 22-24,26,28$ & & & \\
\hline Pagurus excavatus & $\mathrm{A}, \mathrm{B}, \mathrm{C}, 22-25,27,28$ & Turritella communis & $\mathrm{A}, \mathrm{B}, \mathrm{C}$ & & & \\
\hline Pecten jacobaeus & $\mathrm{B}, 25$ & Veretilum cynomorium & $\mathrm{A}, \mathrm{B}, \mathrm{C}, 25$ & & & \\
\hline Phallusia mamillata & $\mathrm{A}, \mathrm{B}, \mathrm{C}, 22-24,26,27$ & & & & & \\
\hline \multicolumn{7}{|c|}{ UPPER SUBLITTORAL ZONE } \\
\hline IPA, CCSA and HP & & & & & WGFS and BGIS & \\
\hline Arbacia lixula & 23 & Maja squinado & \multicolumn{2}{|c|}{$24,25,28$} & Acanthocardia tuberculata & 22 \\
\hline Axinella cannabina & 26 & Marthasterias glacialis & \multicolumn{2}{|l|}{24} & Anadara corbuloides & 25 \\
\hline Bolma rugosa & 26 & Modiolus barbatus & \multicolumn{2}{|l|}{25} & Anadara diluvii & $\mathrm{A}$ \\
\hline Dromia personata & 28 & Pisidia longimana & \multicolumn{2}{|l|}{25,26} & Scrobicularia plana & 25 \\
\hline Echinaster sepositus & 27 & Psammechinus microtuberculatus & \multicolumn{2}{|l|}{23} & & \\
\hline Euspira guillemini & 25 & Scalarispongia scalaris & $\mathrm{A}, \mathrm{B}, \mathrm{C}$ & & & \\
\hline Galathea strigosa & 28 & Sphaerechinus granularis & $22,23,26$ & $5-28$ & & \\
\hline Maja crispata & $22,24,25$ & Spondylus gaederopus & 28 & & & \\
\hline & & EUBUS & & & & \\
\hline Antendon mediterraneus & 26 & Dardanus arrosor & $22,24,2$ & & & \\
\hline Astropecten irregularis & $\mathrm{A}, \mathrm{B}, \mathrm{C}, 22-26$ & Liocarcinus depurator & $\mathrm{A}, \mathrm{B}, \mathrm{C}, 26$ & 6,28 & & \\
\hline Atrina pectinata & $\mathrm{A}, \mathrm{B}, \mathrm{C}$ & Macropodia longirostris & $22-26$ & & & \\
\hline Calappa granulata & 28 & Maja goltziana & 28 & & & \\
\hline Calliactis parasitica & $\mathrm{A}, \mathrm{B}, \mathrm{C}, 22-28$ & Pleurobranchaea meckeli & 24 & & & \\
\hline Cerianthus membranaceus & $\mathrm{A}$ & Squilla mantis & $\mathrm{A}, \mathrm{B}, \mathrm{C}, 22$ & $2-28$ & & \\
\hline Cerithium vulgatum & $23,24,26-28$ & Tethya aurantium & $\mathrm{C}$ & & & \\
\hline Chaetaster longipes & 28 & Thracia pubescens & $\mathrm{A}$ & & & \\
\hline
\end{tabular}


multifaceted significance of such knowledge, the available information is very limited; the interest up to date has focused on commercial invertebrate (mostly crustaceans and molluscs) and fish discards, giving occasionally some information on the non-commercial species fraction $[2,7$, 14]. In the very few comprehensive studies of the noncommercial invertebrate discards [3, 24], high percentage contributions of other groups, such as echinoderms and cnidarians were recorded; however, the overall synthesis of invertebrate discards seems to differ between geographic areas $[2,24]$.

The comparison of discarded taxa composition found in this study with the overall composition of benthic taxa in the Hellenic Seas and specifically the commercially exploited ones [25, 26] showed that molluscs and crustaceans prevail in all cases. These two taxa are the most important components of benthic assemblages in sublittoral trawlable bottoms of the Mediterranean [27, 28]. Echinoderms showed a quite high per cent species richness in the discards, yet not as high as in trawl catch [2] or trammel nets [3] from other areas.

Although the species richness of discarded invertebrates cannot be a proper predictor of the local benthic diversity, several issues concerning benthic community structure and distribution in the studied area can be considered on the basis of the discards faunal composition. Thus, certain benthic biocoenoses were identified, though not clearly delimited, in the studied area through the presence of characteristic species:

(i) Coastal detritic (CD) and muddy detritic biocoenoses (MD) were detected all over the sampling area. All species typical of the CD biocoenosis such as Flexopecten flexuosus, Pecten jacobeus, Holothuria tubulosa, Phallusia mamilata were represented in Ssf areas and several on the Msf stations. This shows that coastal detritic bottoms cover a large part of the sublittoral zone (both upper and lower) in this area. The finding of Nephrops norvegicus in area C of Msf confirms the presence of the MD biocoenosis in the deepest parts of the Gulf.

(ii) The Coastal terrigenous muds biocoenosis (CTM) was mostly recognized in the discards of Msf, which was conducted in the western part of the Gulf and particularly in north-western station group A; this biocoenosis is commonly settled on clayey mud of fluvial origin, which is the prevailing sediment in this part of the Gulf, where the river outflow is more influential [31]. The characteristic species Turitella communis, Alcyonium palmatum, Goneplax rhomboides, and Aphrodita aculeata occurred in all Msf and sporadically in Ssf stations. The species Parapenaeus longirostris and Funiculina quadrangularis confirmed the existence of the Bathyal muds biocoenosis (BM) in the deepest part of the study area (Msf area $\mathrm{C}$ ); the former species, although present in all Msf A and B stations, was not included in the discards of area $\mathrm{C}$ since it replaced Melicertus kerathurus as a target species and thus was not discarded at all.

(iii) Several plant dominated communities were recognized in the Ssf discards, i.e. in the shallower parts of the Gulf, both in the eastern and the western part. This is in accordance with the distribution of such communities in the upper sublittoral zone. Besides the photophilous algae (IPA) and Posidonia meadows (HP) biocoenoses, the sciaphilous algae biocoenosis (CCSA) often thrives in well shaded sites of this zone, regardless of depth [29].

The clear differentiation in discard species composition observed between the two fisheries, reflects the different habitats in which fishery gears were deployed: Msf trawls were mostly working in depths of 33-95 $\mathrm{m}$ and the discarded species were typical of the clayey assemblages found in the lower sublittoral and upper bathyal zones. Trammel and gill nets were set in shallower waters of the upper sublittoral zone (mostly from 3 to $40 \mathrm{~m}$ ), often near areas covered with vegetation. This is why many of the Ssf discards were species typical of algal and Posidonia dominated communities, sandy bottoms and lagoon communities. Depth and sediment type are the main factors structuring benthic communities on the soft trawlable substrata of the continental shelf $[20,30]$. Thus, they were expected to have an impact on the composition of the discarded material, which reflects the epibenthic community structure. As determinant factors of discards diversity in Msf, previous researchers indicate either depth [7] or season [3]. In our case it is difficult to consider such relationships since the two factors have not been studied separately.

The relative homogeneity in the bottom and community structure in the Msf areas is probably the reason for the lower diversity of discards (in comparison with the Ssf) and the higher similarity among them in terms of discard species composition. On the other hand, the seven areas of Ssf were more diverse in discarded invertebrates, due to the greater variety of adjacent habitats and established communities. Due to particular environmental conditions, stations 25 and 28 were differentiated from the rest Ssf stations. The former is the only station located in Thessaloniki Bay, where the impact of the river output and urban pollution is higher, and the latter in the eastern part of the outer Gulf, along which the cleaner and oligotrophic Aegean waters entering Thermaikos Gulf moves northwards [31].

All the above observations become more important under the scope of the strong correlation existing between the epifaunal benthic diversity and community structure, and the demersal fish assemblages in the Mediterranean Sea, which stresses the need for an ecosystem-based demersal resources management [4]. Moreover, some other discarded sessile species, such as sponges and ascidians, are of increased importance since they contribute as ecosystem engineers to the habitat complexity and benthic community structuring in the North Aegean Sea [32].

The results of this study show that the species composition of discarded invertebrates can be suitable predictor of benthic community structure and distribution in a given area. Such biological background data could prove extremely useful in the development of ecological footprint models for marine areas under intense anthropogenic pressure (e.g. overfishing), which in turn may serve as biomonitoring tools. Although in the last decade, an increasing number of models have been proposed for the monitoring of biological systems [33], those do not seem to meet the requirements of a common for all systems and user-friendly practice. Efforts to investigate the applicability of models in benthic marine 
ecosystem health monitoring are particularly compelling today. This is because the benthic ecosystem is inhabited by more than $85 \%$ of the global marine biodiversity and yet its complexity has not been studied at all levels of organization [34]. The necessity for development of models appropriate for the monitoring of complex benthic systems has already been indicated by various authors [35].

\section{ACKNOWLEDGEMENT}

The authors would like to thank Thanos Dailianis for artwork preparation.

\section{REFERENCES}

[1] Davis RWD, Cripps SJ, Nickson A, Porter G. Defining and estimating global marine fisheries bycatch. Mar Policy 2009; 33: 661-72.

[2] Sanchez P, Sartor P, Recasens L, et al. Trawl catch composition during different fishing intensity periods in two Mediterranean demersal fishing grounds. Sci Mar 2007; 71: 765-73.

[3] Concalves JMS, Bentes R, Coehlo R, et al. Non-commercial invertebrate discards in an experimental trammel net fishery. Fisheries Manag Ecol 2008; 15: 199-210.

[4] Colloca F, Cardinale M, Belluscio A, Ardizzone G. Patterns of distribution and diversity of demersal assemblages in the central Mediterranean sea. Estuar Coast Shelf S 2003; 56: 469-80.

[5] Reiss H, Degraer S, Duineveld GCA, et al. Spatial patterns of infauna, epifauna, and demersal fish communities in the North Sea. ICES J Mar Sci 2009; 67: 278-93.

[6] Malaquias MAE, Bentes L, Erzini K, Borges TC. Molluscan diversity caught by trawling fisheries: a case study in southern Portugal. Fisheries Manag Ecol 2006; 13: 39-45.

[7] Sanchez P, Demestre M, Martin P. Characterization of the discards generated by bottom trawling in the northwestern Mediterranean. Fish Res 2004; 67: 71-80.

[8] FAO. Increasing the contribution of small-scale fisheries to poverty alleviation and food security. FAO Technical Guidelines for Responsible Fisheries. 2005; No. 10, 1-97.

[9] Batista MJ, Teixeira C, Cabral H. Catches of target species and bycatches of an artisanal fishery: The case study of a trammel net fishery in the Portuguese coast. Fish Res 2009; 100: 167-77.

[10] Hellenic Statistical Authority [Homepage on the Internet]. Quantity of Fish Landed by Fishing Area and Fishing gear; 2009 [cited 2011 Feb 20]. Available from: www.statistics.gr.

[11] Stergiou KI, Politou CY, Christou ED, Petrakis G. Selectivity experiments in the NE Mediterranean: the effect of trawl codend mesh size on species diversity and discards. ICES J Mar Sci 1997; 54: 774-86.

[12] Lamprakis MK, Kallianiotis AA, Moutopoulos DK, Stergiou KI. Weight-length relationships of fishes discarded by trawlers in the North Aegean Sea. Acta Ichthyol Piscat 2003; 13: 145-51.

[13] Machias A, Maiorano P, Vassilopoulou V, Papaconstantinou C, Tursi A, Tsimenidis N. Sizes of discarded commercial species in the eastern-central Mediterranean Sea. Fish Res 2004; 66: 213-22.

[14] Machias A, Vassilopoulou V, Vatsos D, et al. Bottom trawl discards in the northeastern Mediterranean Sea. Fish Res 2001; 53: 181-95.

[15] Kallianiotis A, Vidoris P, Sylaios G. Fish species assemblages and geographical sub-areas in the Northern Aegean Sea, Greece. Fish Res 2004; 68: 171-87.
[16] Zarkanellas AJ, Kattoulas ME. The ecology of benthos in the Gulf of Thermaikos, Greece. I. environmental conditions and benthic biotic indices. Mar Ecol 1982; 3: 21-39.

[17] Antoniadou C, Krestenitis Y, Chintiroglou C. Structure of the "Amphioxus sand" community in Thermaikos Bay (Eastern Mediterranean). Fresen Environ Bull 2004; 13: 1122-8.

[18] Karageorgis AP, Anagnostou CL. Particulate matter spatialtemporal distribution and associated surface sediment properties: Thermaikos Gulf and Sporades Basin, NW Aegean Sea. Cont Shelf Res 2001; 21: 2141-53.

[19] Clarke KR, Gorley NR. PRIMER v6: User manual/tutorial, Plymouth: Primer-E Ltd; 2006.

[20] Pérès JM, Picard J. Nouveau manuel de bionomie benthique de la mer Méditerranée. Recl Travaux St Mar End 1964; 47: 1-137.

[21] Augier H. Inventory and classification of marine benthic biocenoses of the Mediterranean. Nature and Environment Series, 25. Strasbourg: Conseil de l' Europe; 1982.

[22] Aguilar R, Pastor X, De Pablo MJ. Habitats in danger. Madrid: Oceana; 2006.

[23] Costa ME, Erzini K, Borges TR. Bycatch of crustacean and fish bottom trawl fisheries from southern Portugal (Algarve). Sci Mar 2008; 72: 801-14.

[24] Probert PK, McKnight DG, Grove SL. Benthic invertebrate bycatch from a deep-water trawl fishery, Chatham Rise, New Zealand. Aquat Conserv 1997; 7: 27-40.

[25] Zenetos A, Arvanitidis C, Thessalou-Legaki M, Simboura N. Zoobenthos-Soft bottom fauna. In: Papathanassiou E, Zenetos A, Eds. State of the Hellenic marine environment. Athens, Hellenic Centre for Marine Research 2005; 236-46.

[26] Chintiroglou C, Antoniadou C, Vafidis D, Koutsoubas D. In: Papathanassiou E, Zenetos A, Eds. State of the Hellenic marine environment. Athens, Hellenic Centre for Marine Research 2005; 247-53.

[27] Serrano A, Sánchez F, García-Castrillo G. Epibenthic communities of trawlable grounds of the Cantabrian Sea. Sci Mar 2006, 70SI, 149-59.

[28] García Muñoz JE, Manjón-Cabeza ME, García Raso JE. Decapod crustacean assemblages from littoral bottoms of the Alboran Sea (Spain, west Mediterranean Sea): spatial and temporal variability. Sci Mar 2008; 72: 437-49.

[29] Uriz M, Rosell D, Martin D. The sponge population of the Cabrera Archipelago (Balearic Islands): characteristics, distribution and abundance of the most representative species. Mar Ecol 1992; 13: 101-17.

[30] Abad E, Preciado I, Serrano A, Baro J. Demersal and epibenthic assemblages of trawlable grounds in the northern Alboran Sea (western Mediterranean). Sci Mar 2007; 71: 513-24.

[31] Poulos SE, Chronis GT, Collins MB, Lykousis V. Thermaikos Gulf Coastal system, NW Aegean Sea: an overview of water/sediment fluxes in relation to air-land; ocean interactions and human activities. J Marine Syst 2000; 25: 47-76.

[32] Voultsiadou E, Kyrodimou M, Antoniadou C, Vafidis D. Sponge epibionts on ecosystem engineering ascidians: The case of Microcosmus sabatieri. Estuar Coast Shelf S 2010; 86: 598-606.

[33] Jorgensen SE. Overview of the model types available for development of ecological models. Ecol Model 2008; 215: 3-9.

[34] Zacharias MA, Roff JC. A hierarchical ecological approach to conserving marine biodiversity. Conserv Biol 2000; 14: 1327-34.

[35] Chintiroglou CC, Antoniadou C. Polychaetes/Amphipods Index. In: Jorgensen SE, Fath BD, Eds. Ecological Indicators, Encyclopedia of Ecology. Oxford: Elsevier 2008; vol. 4: 2868-72.

This is an open access article licensed under the terms of the Creative Commons Attribution Non-Commercial License (http://creativecommons.org/licenses/by$\mathrm{nc} / 3.0 /$ ), which permits unrestricted, non-commercial use, distribution and reproduction in any medium, provided the work is properly cited. 